\title{
Molecular Docking and Molecular Dynamic Study of two Viral Proteins associated with SARS-CoV-2 with Ivermectin
}

\author{
Lenin A. González-Paz ${ }^{1,}$ *, Carla A. Lossada², Luis S. Moncayo ${ }^{3}$, Freddy Romero ${ }^{4}$, J. L. \\ Paz $^{5}$, Joan Vera-Villalobos ${ }^{6}$, Aleivi E. Pérez ${ }^{7}$, Ernesto San-Blas ${ }^{8}$, Ysaias J. Alvarado ${ }^{2}$ * \\ ${ }^{1}$ Laboratorio de Genética y Biología Molecular (L.G.B.M). Departamento de Biología. Facultad Experimental de Ciencias (F.E.C). \\ Universidad del Zulia (L.U.Z). Maracaibo, Republica Bolivariana de Venezuela. \\ ${ }^{2}$ Laboratorio de Caracterización Molecular y Biomolecular- Centro de Investigación y Tecnología de Materiales (CITeMA)- Instituto \\ Venezolano de Investigaciones Científicas (I.V.I.C)- Zulia. Maracaibo, Republica Bolivariana de Venezuela. \\ ${ }^{3}$ Universidad Católica de Cuenca, Unidad Académica de Salud y Bienestar. Farmacia, Laboratorio de Microbiología. Ecuador. \\ ${ }^{4}$ Center for Traslational Medicine. Thomas Jefferson University. Philadelphia. PA 19107, USA. \\ ${ }^{5}$ Departamento de Física. Escuela Politécnica Nacional, Ladrón de Guevara. E11-253,170517; Quito- Ecuador. \\ ${ }^{6}$ Escuela Superior Politécnica del Litoral, Facultad de Ciencias Naturales y Matemáticas. Departamento de Química y Ciencias Ambientales. \\ Laboratorio de Análisis Químico Instrumental (LAQUINS). \\ ${ }^{7}$ Laboratorio de Microbiología General (L.M.G). Departamento de Biología. Facultad Experimental de Ciencias (F.E.C). Universidad del \\ Zulia (L.U.Z). Maracaibo, Republica Bolivariana de Venezuela. \\ ${ }^{8}$ Laboratorio de Protección Vegetal, Centro de Estudios Botánicos y Agroforestales, Instituto Venezolano de Investigaciones Científicas \\ (I.V.I.C)- Zulia. Maracaibo, Republica Bolivariana de Venezuela.
}

*Correspondence: lgonzalezpaz@gmail.com; Tel.: +584246201133 (VEN).

*Correspondence: alvaradoysaias@ gmail.com; Tel.: +584265604328 (VEN)

\section{Abstract}

The global pandemic caused by the new SARS-COV-2 coronavirus makes it necessary to search for drugs for its control. Within of this research it has been known that the ivermectin drug, a FDA-approved drugs which is formulated as an 80:20 mixture of ivermectin B1a and B1b and used commonly for parasitic infections, has an inhibitory effect on viruses, includes SARS-COV-2 at in vitro level. In the particular case of SARS-COV-2 its mechanism of action remains elusive and controversial. Interestingly, the energy of interaction of ivermectin with any of the proteins the SARS-CoV-2 and the possible structural alterations at the protein level that this drug can cause have not been reported. In this sense, we carried out a bioinformatics study with docking strategies and molecular dynamics to predict the binding and disturbance induced by ivermectin in proteins associated with SARS-CoV-2. We use DockThor and Molegro docking scores. The DockThor server and myPresto software were used to build complexes and dynamics studies, respectively. The results obtained suggested that ivermectin is capable of docking with the 3CL protease and the HR2 domain, and may promote structural changes in these proteins by inducing unfolding/folding. Specifically, ivermectin brings protease to a significantly more deployed conformational state and the HR2 domain to a more compact state compared to the native state. Finally, it is shown that B1a and B1b macrocyclic lactones have a behavior different from to each target protein. These results suggest a possible inhibitory effect against SARS-CoV-2 due to a synergistic role of this drug to spontaneously bind to two important proteins involve in the proliferation of this virus. However, more studies are required on this possible mechanism of action.

Key words: SARS-COV-2, folding/unfolding, antiparasitic, molecular docking, molecular dynamics, COVID-19, Ivermectin. 


\section{Introduction}

The first SARS-CoV-2 Coronavirus case was reported in December 2019 in Wuhan City, P.R. China (Xu et al., 2020), and in early April, cases totaled 1,133,758 confirmed cases and 62,784 deaths worldwide (WHO, 2020) therefore, the rapid approval of a vaccine not yet available to date is required (Jiang et al., 2020). Study of FDA-approved drugs with antiviral activity against related viruses has been recommended as a promising alternative (Chang et al., 2020; Contini, 2020). It was recently reported that ivermectin, drug (80:20 mixture of two homologous macrocyclic lactones B1a and B1b), a FDA-approved drugs used for parasitic infections, has an inhibitory effect on viruses, includes SARS-COV-2 at in vitro level, although the mechanism by which it has inhibitory activity in vitro against the new coronavirus is still unclear and further studies have been recommended (Caly et al., 2020). However, other actions of ivermectin have been reported such as the ability to inhibit virus replication by enzyme inhibition (Mastrangelo et al., 2012), by inactivating enzymes similar to those described in SARS-CoV required for viral replication (Tong, 2009). Additionally, several theoretical studies have already proposed several proteins associated with SARSCOV-2 as possible targets with therapeutic interest, and thousands of dockings have been performed with various compounds (Chang et al.,2020; Liu et al., 2020; Khaerunnisa et al., 2020; Mohammad et al., 2020; Qamar et al., 2020; Sharma and Kaur, 2020; Chandel et al., 2020; Adem et al., 2020; Gentile et al., 2020; Sun et al., 2020), and in silico studies have been done between ivermectin and related viruses (Mastrangelo et al., 2012), but theoretical study about the energetic of interaction of ivermectin with any of the proteins the SARS-CoV2 as well as the possible structural alterations at the protein level that this drug can cause have not been reported.

There are few reports on the use of the MolDock docking algorithm with proteins associated with SARS-CoV-2 (Adem et al., 2020), an extension of docking based on linear part potential 
(PLP) with improvements for docking precision (Thomsen and Christensen, 2006; Wang, 2020). This is the first bioinformatics study on the structural alteration that ivermectin can induce in viral proteins associated with SARS-CoV-2 (Alamri et al., 2020). Therefore, we conducted a study with various molecular docking algorithms, including MolDock, to study the interaction of the two ivermectins $\mathrm{B} 1 \mathrm{a}$ and $\mathrm{B} 1 \mathrm{~b}$ present in the drug Ivermectin against various proteins associated with SARS -CoV-2.

\section{Methods}

Docking Screening: In this study, the following structures associated with SARS-CoV-2 were considered and obtained from the RCSB protein database: 3CL protease (PDB ID: 6LU7) (Chang et al., 2020), 2019-nCoV HR2 domain (PDB: 6lvn), S2 2019-nCoV subunit (PDB: 61xt), 2019-nCoV spike glycoprotein (PDB: 6vsb), 2019-nCoV chimeric receptor binding domain (PDB: 6vw1), and SARS CoV-2 endoribonuclease NSP15 (PDB: 6vww) (Mohammad et al. Al., 2020). The 2D structures of the compounds ivermectin B1a (CID_6321424) and ivermectin B1b (CID_6321425) were obtained from PubChem in SDF format, and the SMILES online converter was used (https://cactus.nci.nih.gov / translate /) to get a PDB format. To simulate ligand-protein binding the complexes were constructed in DockThor (https://dockthor.lncc.br/v2/) using the flexibility algorithm and blind docking (Dos Santos et al., 2020). The most favored position was analyzed with MMV_2019_7.0.0, calculating the MolDock and Rerank functions (Thomsen and Christensen, 2006). We also used myPresto docking to couple selected drugs to protease in the default configuration (Kasahara et al., 2016).

Molecular dynamics (MD) simulation: MD simulations were performed for a docking coup to study the relative stability of the ligand in the complex and to sample a set of conformations as a function of time. An MD simulation consisted of a copy of the SARS-COV-2-associated 
protein, a copy of the docked ligand, and $50 \mathrm{Na}+$ and $\mathrm{Cl}-$ ions. The system was neutralized. For a protein-ligand complex, the MD system was first relaxed through minimization procedures as recommended (Wang, 2020). All MD simulations and additional configurations were performed with the myPresto program.

Complementary Analysis: The Molinspiration server was used for the bioactivity calculations (Cheminformatics, 2020; Reena Roy et al., 2020) and tools the Molecular Modeling Group of the SIB | Swiss Institute of Bioinformatics (Zoete et al., 2016; Daina et al., 2017).

\section{Results and Discussion}

In Table 1, we list the results of the docking of ivermectin B1a_CID_6321424 and ivermectin B1b_CID_6321425 with the newly released protein structures of SARS-CoV-2. These scores, represent the relative binding affinity obtained with DockThor (Dock Score) and Molegro (MolDock, Rerank and Plants Scores). All scoring functions predicted at least two thermodynamically favorable docking between ivermectins (B1a and B1b) and the proteins studied. The DockT and Plants scoring functions were the only scoring functions that calculated favorable dockings in all cases. While the MolDock and Rerank functions only predicted a thermodynamically favorable docking between lactones and the 3CL-protease (PDB: 6lu7) and 2019-nCoV HR2 domain (PDB: 6lvn) proteins of $-11.939 /-7.037 \mathrm{kcal} / \mathrm{mol}$ and -9.971 / -26.034 kcal/mol in MolDock, and -16.622 / -8.722 kcal/mol and -12.062 / $26.145 \mathrm{kcal} / \mathrm{mol}$ in Rerank for ivermectin B1a and B1b, respectively.

It is important to note that all scoring functions predicted that the most favorable docking is likely between ivermectins and these two only proteins. These results seen in Table 1 are interesting if we consider that the MolDock docking algorithm, which consists of an extension of the PLP that includes new hydrogen bonds and electrostatic terms, and tends to improve the docking precision (Thomsen and Christensen, 2006; Wang, 2020). Interestingly, all the 
algorithms considered, except the DockT Score, predicted that ivermectin B1a has a higher affinity for the 3CL-protease, while ivermectin B1b has a higher affinity for the 2019-nCoV HR2 domain. A result that supports the combined use that has been given to these molecules (Yazwinski et al., 1981) due to the fact that in terms of docking a synergistic action is observed in this study. Although it is not known if these results are related to the antiviral activity that has been reported (Varghese et al., 2016; Caly et al., 2020). But these dockings do not fail to attract attention due to the fact that the 3CL-protease and the 2019-nCoV HR2 domain could be considered as the most important structures associated with SARS-COV-2 in the research and development of treatment strategies (Chang et al., 2020; Mohammad et al., 2020; Adem et al., 2020; Alamri et al., 2020; Arya et al., 2020; Bzowka et al., 2020; Chandel et al., 2020; Choudhary et al., 2020; Contini, 2020; Elshabrawy et al., 2012; Gentile et al., 2020; Sekhar, 2020; Sharma et al., 2020; Sun et al., 2020; Qamar et al., 2020; Wang, 2020; Xia et al., 2020; Zhang et al., 2020). We therefore choose the 3CL-protease and the 2019$\mathrm{nCoV}$ HR2 domain for further investigation with the filter MM. Making a comparative analysis between ivermectin B1a and B1b (see Table 1, Figure 1 - 2). 
Table 1. The results of the virtual examination were performed on crystalline structures of the proteins associated with SARS-COV-2. Showing the punctuation functions of docking with ivermectin B1a and B1b, two homologous lactones ancient antiparasitic compounds approved by the FDA and which have given promising results in vitro against SARS-COV-2. The docking with the best scores are highlighted in bold.

\begin{tabular}{|c|c|c|c|c|c|}
\hline \multirow[b]{2}{*}{$P D B$} & \multicolumn{5}{|c|}{ Ivermectin B1a_CID_6321424 / Ivermectin B1b_CID_6321425* } \\
\hline & $\begin{array}{c}\text { DockT Score } \\
\text { (kcal/mol) }\end{array}$ & $\begin{array}{l}\text { MolDock Score } \\
(\text { kcal/mol })\end{array}$ & $\begin{array}{l}\text { Rerank Score } \\
(\text { kcal/mol })\end{array}$ & $\begin{array}{l}\text { Plants Score } \\
\text { (kcal/mol) }\end{array}$ & Interactions \\
\hline $6 \mathrm{vsb}$ & $-8.325 /-8.373$ & $5.430 / 5.905$ & $0.786 / 0.311$ & $-23.713 /-24.210$ & Asp950(A) $)^{\mathrm{HB}} / \mathrm{Asp} 950(\mathrm{~A})^{\mathrm{HB}}$ \\
\hline $6 l u 7$ & $-8.642 /-8.287$ & $-11.939 /-7.037$ & -16.622 / -8.722 & $-39.648 /-31.570$ & $\begin{array}{c}\text { Lys }^{\mathrm{HB}}, \text { Leu } 282^{\mathrm{HB}}, \text { Ser } 284^{\mathrm{SI}} / \mathrm{Lys}^{\mathrm{HB}}, \\
\text { Arg } 4^{\mathrm{HB}-\mathrm{SI}}\end{array}$ \\
\hline $6 \mathrm{vw} 1$ & $-8.367 /-7.833$ & $15.880 / 30.797$ & $9.617 / 20.543$ & $-19.158 /-15.138$ & $\operatorname{Asn} 586(\mathrm{~A})^{\mathrm{HB}} / \mathrm{ND}$ \\
\hline $6 \mathrm{vww}$ & $-7.776 /-7.526$ & $17.410 / 13.048$ & $8.101 / 7.551$ & $-24.404 /-23.025$ & $\begin{array}{l}\text { Asn140(B) }{ }^{\mathrm{HB}}, \operatorname{Asp} 79(B)^{\mathrm{HB}} \\
\text { Asp184(B) } \\
\text { HB } / \text { Gln347(B) }{ }^{\mathrm{HB}}\end{array}$ \\
\hline
\end{tabular}

*, Ivermectin is a lactone macrocyclic type drug consisting of an 80:20 mixture of ivermectin B1a and B1b; PDB, Protein Data Bank; 6LU7, 3CL-protease; 6lvn, 2019-nCoV HR2 domain; 6lxt, 2019-nCoV S2 subunit; 6vsb, 2019-nCoV spike glycoprotein; 6vw1, 2019-nCoV chimeric receptor-binding domain; 6vww, NSP15 Endoribonuclease from SARS-CoV-2; ${ }^{\mathrm{HB}}$, hydrogen bonds; ${ }^{\text {SI }}$, Steric Interactions; ND; not determined. 

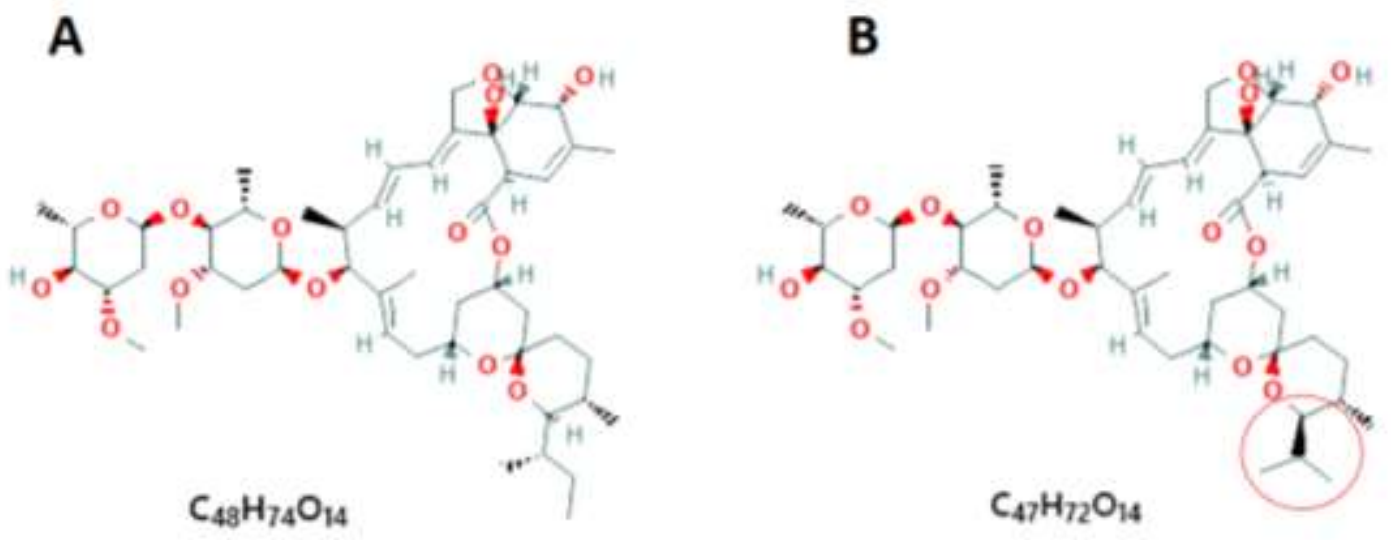

Figure 1. Visualization of the antiparasitic compounds approved by the FDA considered in this study. A) ivermectin_B1a_CID_6321424 and B) ivermectin_B1b_CID_6321425.

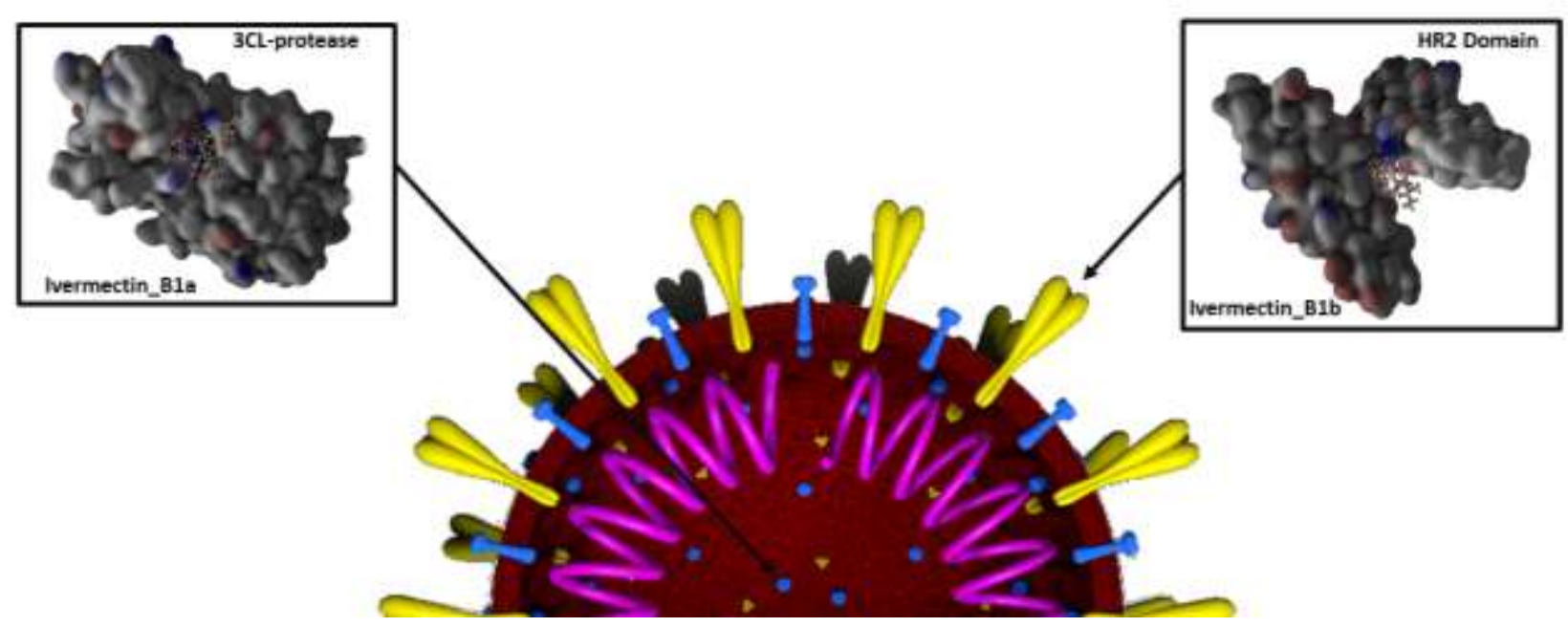

Figure 2. An illustrative view showing the functional and structural proteins of SARS-CoV-2, the 3CL-protease and HR2-domain, respectively. Based on molecular docking, they are shown in boxes the type of ivermectin suggested with the highest activity in terms of the best docking functions. 
Table 2. Prediction of the partition coefficient and bioactivity scores with the Molinspiration server and tools the Molecular Modeling Group of the SIB | Swiss Institute of Bioinformatics. Before calculating the trajectories of the different positions of the atoms of the ligand-protein complexes as a function of time, the bioaccumulation and bioactivity of the ligands was determined.

\begin{tabular}{cccccc}
\hline Compounds & MW $(\mathrm{g} / \mathrm{mol})$ & $\log \boldsymbol{K}_{\mathrm{ow}}$ & $\boldsymbol{P S}$ Score & E Score & $K_{l}$ \\
\hline Ivermectin B1a_CID_6321424 & 875.09 & 4.58 & -1.89 & -2.53 & + \\
Ivermectin B1b_CID_6321425 & 861.07 & 4.08 & -1.68 & -2.39 & + \\
\hline
\end{tabular}

$\log K_{\mathrm{ow}}, 2$ - 5 (Bhal, 2007); P Score, protease inhibitor score (-0.50 - 1.50) Cheminformatics, 2020; E Score, enzyme inhibitor score (-0.50 - 1.50) Cheminformatics, 2020; $K_{l}$, similarity score with ligands for kinase (Identity= 0.98; Zoete et al., 2016).

After studying the molecular properties and structural characteristics of these compounds, it was determined that the ligands have a good partition coefficient $\left(\log K_{\text {ow }}: 4.08-4.58\right)$, and these results suggest that the studied compounds are capable of bioaccumulating with a high coefficient (Bhal, 2007) (Table 2). In addition, the potential bioactivity of these compounds was studied, and it was found that the antiparasitics studied do not present typical characteristics of enzyme inhibitors in general, including protease inhibitors based on what was reported in the database considered (Cheminformatics, 2020) (see Table 2). We also examined the similarity of the compounds studied with the tools the Molecular Modeling Group of the SIB | Swiss Institute of Bioinformatics, and although we found once again that ivermectins do not possess the structural characteristics and molecular properties of the model compounds for enzyme inhibition as described for other viruses (Mastrangelo et al., 2012), and we were only able to trace their activity as a substrate for P-glycoproteins already described and predicted (Caly et al., 2020; Daina et al., 2017). It is interesting to note that it presents a high structural similarity score with ligands for kinase (Identity $=0.98$; Zoete et al., 2016), a comparative computational analysis that 
should not be ignored because it provides information on a possible mechanism of action of ivermectins, because it was shown that the signaling pathways of signal-related extracellular kinase (ERK1/2), N-terminal c-Jun kinase (JNK), mitogen-activated protein kinase p38 (MAPK), and phosphatidylinositol 3'-kinase (PI3K)/Akt are important for the establishment of SARS-CoV (Mizutani et al., 2004; Mizutani et al., 2005). As well as protein kinase R (PKR) and PKR-like endoplasmic reticulum kinase (PERK) (Krähling et al., 2009). In fact, various compounds capable of blocking the ACE2 receptor required by SARS-CoV-2 have been identified by inhibiting the associated Abl kinase. Like what was described against the dengue virus, and similarly against MERS-CoV, by inhibiting ACE2, by inactivating Inositol hexakisphosphate kinase (IP6K) and Akt signaling (Choudhary et al., 2020; Coleman et al., 2016). Interesting results because, like other viruses, SARS-CoV-2 infects lung alveolar epithelial cells using receptor-mediated endocytosis through the angiotensin II converting enzyme (ACE2) as the input receptor, and the use of supercomputers and intelligence Artificial drugs have also predicted that drugs associated with AP2-associated protein kinase 1 (AAK1) that disrupts these proteins may inhibit viral entry into target cells (Velavan and Meyer, 2020). In addition to the fact that antimalarial drugs such as chloroquine recommended for the treatment of COVID-19 (Chang et al., 2020; Devaux et al., 2020) have favorable thermodynamic docking energies for enzymes such as 3CL protease (Gautret et al ., 2020; Wang et al., 2020; Mohammad et al., 2020, Gonzalez-Paz et al., 2020) and are capable of inactivating MAPK as well as the SB203580 (p38 MAPK inhibitor) compound by preventing phosphorylation of the MAPK and viral titers reduced in a dose-dependent manner. It would be interesting to see if the results theoretical of the docking obtained in this study and the similarity between ivermectins with ligands directed to kinases 
could be compared with the observed with antimalarials and likewise extrapolated to SARS-CoV and perhaps other viruses.

Table 3. MD simulations using the myPresto software package. After calculating the trajectories of the different positions of the atoms of the ligand-protein complexes as a function of time.

\begin{tabular}{|c|c|c|c|c|c|c|c|c|}
\hline & \multicolumn{4}{|c|}{ 3CL-protease (PDB: 6lu7)* } & \multicolumn{4}{|c|}{ HR2 domain (PDB: 6lvn)** } \\
\hline Compounds & $\begin{array}{c}\text { - } \\
\text { potential } \\
(\mathrm{kcal} / \mathrm{mol})\end{array}$ & $\begin{array}{c}\text { Time } \\
(p s) /{ }_{E-} \\
\text { potential }\end{array}$ & $\begin{array}{c}\text { E-Total } \\
(\text { kcal/mol })\end{array}$ & $\begin{array}{c}\text { Time } \\
(p s) /{ }_{E-} \\
\text { Total }\end{array}$ & $\begin{array}{c}E- \\
\text { potential } \\
(\mathrm{kcal} / \mathrm{mol})\end{array}$ & $\begin{array}{c}\text { Time } \\
(p s) /{ }_{E-} \\
\text { potential }\end{array}$ & $\begin{array}{c}\text { E-Total } \\
(\mathrm{kcal} / \mathrm{mol})\end{array}$ & $\begin{array}{c}\text { Time } \\
(p s) / \\
\text { E-Total }\end{array}$ \\
\hline $\begin{array}{c}\text { Ivermectin } \\
\text { B1a_CID_6321424 }\end{array}$ & -8960 & 1.8 & -2600 & 3.2 & -4925 & 2.3 & -2050 & 1.6 \\
\hline $\begin{array}{c}\text { Ivermectin } \\
\text { B1b_CID_6321425 }\end{array}$ & -8920 & 1.6 & -2400 & 3.6 & -4820 & 2.7 & -2050 & 2.0 \\
\hline
\end{tabular}

*, E-potential: $-8970 \mathrm{kcal} / \mathrm{mol}$, Time/E-potential: $1.6 \mathrm{ps}$, E-Total: $-2800 \mathrm{kcal} / \mathrm{mol}$, Time/E-Total: $2.8 \mathrm{ps}$; **, E-potential: $-4900 \mathrm{kcal} / \mathrm{mol}$, Time/E-potential: 1 ps, E-Total: $-2300 \mathrm{kcal} / \mathrm{mol}$, Time/E-Total: $1.6 \mathrm{ps}$. Reference values calculated in this study.

After calculating the different positions of the atoms of the ligand-protein complexes as a function of time, to simulate the perturbations of the proteins in the absence and presence of each ligand, we discovered that the two homologous compounds generate thermodynamically stable and similar interactions among themselves in terms of total energy, the interaction of ivermectin_B1a with 3CL-protease $(\Delta \mathrm{G} \leq-2600 \mathrm{kcal} / \mathrm{mol})$ being more favorable than that observed with ivermectin_B1b $(\Delta \mathrm{G} \leq-2400 \mathrm{kcal} / \mathrm{mol})$. It is very important to mention that these magnitudes are close to the values obtained for other docking scores of candidate ligands to viral SARS-CoV-2 proteins (Chang et al., 2020) including values obtained with ligands phytochemicals and antimalaric drugs (Gonzalez-Paz et al., 2020). There was no difference in interaction energy in terms of total energy between lactones and the HR2-domain. On the other 
hand, ivermectin_B1b was the one that caused the greatest disturbance of the thermodynamic stability of the system reflected in a longer time required to reach the most stable structures of minimum energy compared to native proteins, indicating that both drugs affected the stability of the two viral proteins in at least one of the aspects, either potential energy, total energy or the elapsed time to reach the most stable structures. Specifically, 3CL-protease and HR2-domaind in the native state reached their minimum energy structures at $2.8 \mathrm{ps}$ and $1.6 \mathrm{ps}$, in terms of total energy, respectively, but he compounds can induce structural disturbances that affect the thermodynamic stability of the two enzymes, requiring more time according to the simulations to reach the stability of the system (see Table 3). These results show that the compounds considered in this study could theoretically be able to induce important structural disturbances in the target proteins, generating changes in terms of thermodynamic stability at very early stages of docking. In addition, these results correspond to what was observed in the docking, because they show that ivermectin_B1a has a higher affinity for protease while ivermectin_B1b caused the greatest fluctuation in the thermodynamic stability of HR2-domain. A result that again supports the combined use of these molecules for their synergistic action in terms of thermodynamic perturbation of the studied viral protein systems (see Table 3). 

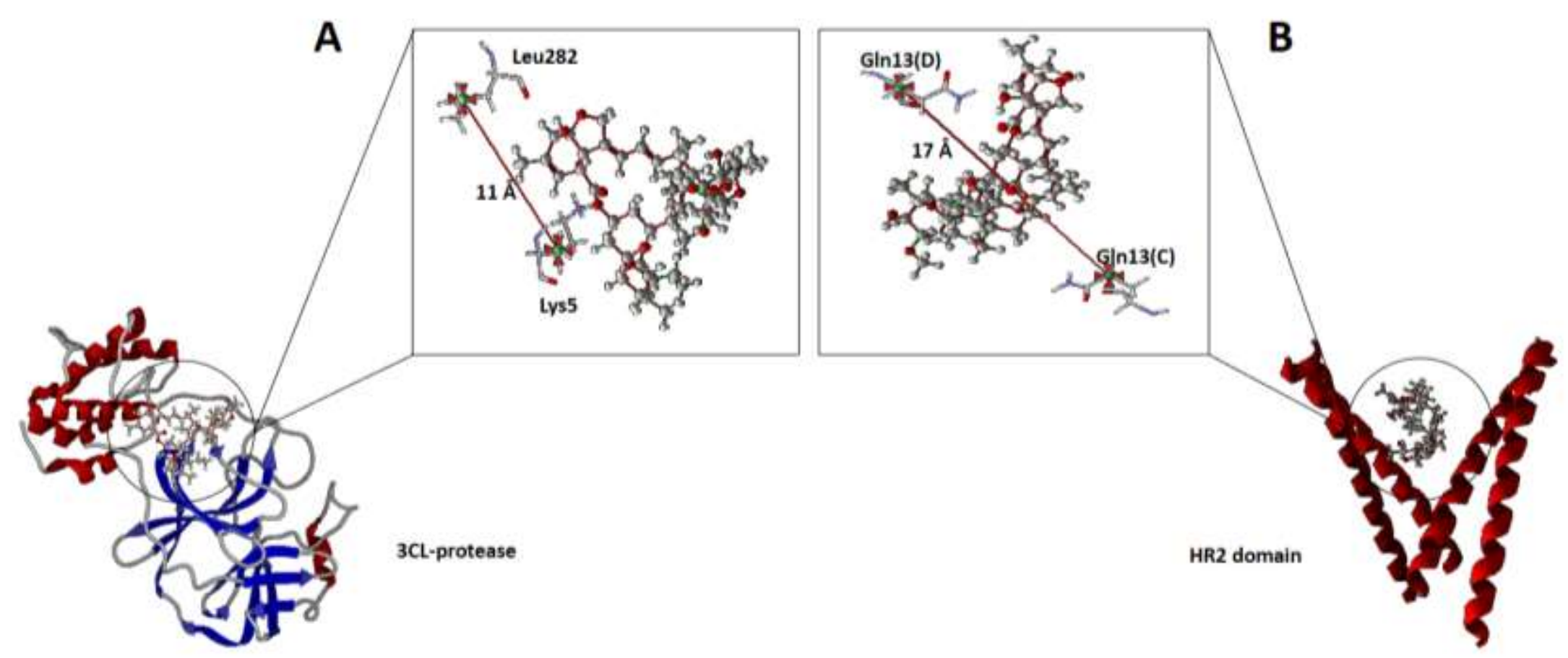

Figure 3. Visualization of the result of docking and location and distance between residues chosen for the MD analyzes are shown. A) 3CL-protease and B) HR2 domain. The figure shows the 3CL protease-ivermectin_B1a and HR2 domainivermectin_B1a docking as reference, respectively. 
Also, we determined that the ligands ivermectin B1a_CID_6321424 and ivermectin B1b_CID_6321425 are capable of inducing structural disturbances in the viral protease and in the HR2 domain using as a reference the distances between the residues of Lys5 and Leu282, and Gln13 (chain D) and Gln13 (chain C) for 3CL-protease and HR2 domain, respectively. Arbitrarily chosen because they are at a distance of approximately $3-4 \AA$ from the ligands (see Figure 3).

Specifically, the studied compounds can induce the deployment of the viral 3CL-protease in very early stages of their interaction, ivermectin_B1a was the compound that induced the greatest structural alteration of the viral protease in terms of deployment in less than 1 ps according to the simulation and was predicted at 2 ps an unfolding of approximately almost $25 \%$. Similar results predicted with ivermectin_B1b but with minor degree unfolding of this protease. In contrast, the results shown that two ivermectins caused the folding of the HR2-domain, with ivermectin_B1a causing the greatest structural change in terms of folding of the macromolecule, reflected by almost $15 \%$ of folding in very early stages of the interaction. Additionally, ivermectin_B1a and ivermectin_B1b caused a fold of approximately $10 \%$ at 2 ps from the simulation, all compared to the native state (see Figure 4).

However, it is unknown whether these microscopic perturbations induced to the viral protease and the HR2 domain are related to biological activity observed both in vitro and in vivo against SARS-CoV-2 to any of the associated viruses. But they are consistent with the predicted alterations in terms of thermodynamic stability and with the best observed docking. The structural compaction induced in the HR2 domain by ivermectins does not fail to attract attention, 
as well as the important deployment of the viral protease because the molecular processes involving these proteins could theoretically be affected in some way. Because compact protein states are known to be very stable in the intracellular environment, which is highly congested by the presence of large amounts of macromolecules (Gomez et al., 2019) and that the unfolding of proteins tends to be associated with the loss of biological activity (Askin et al., 2018). 
A

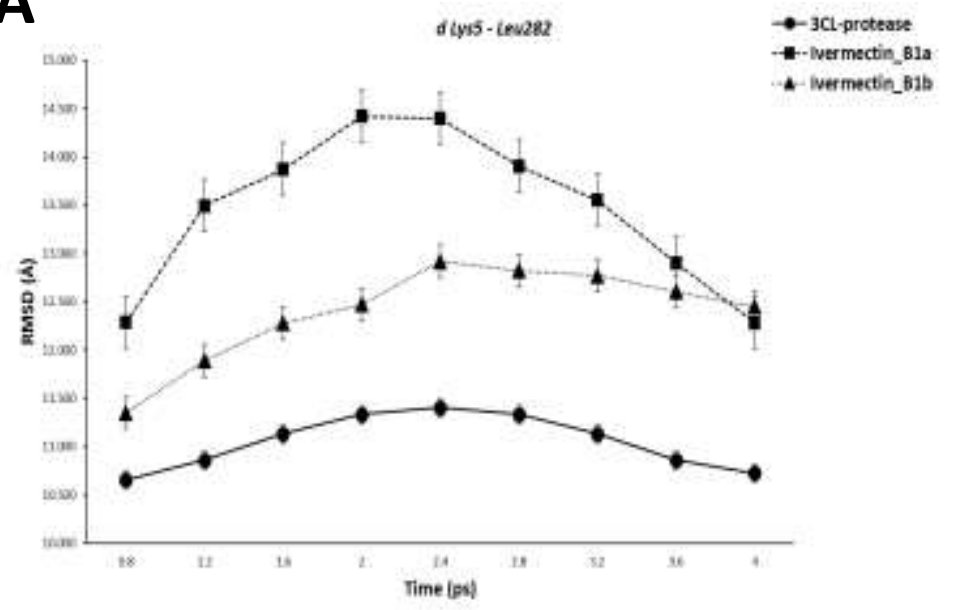

C

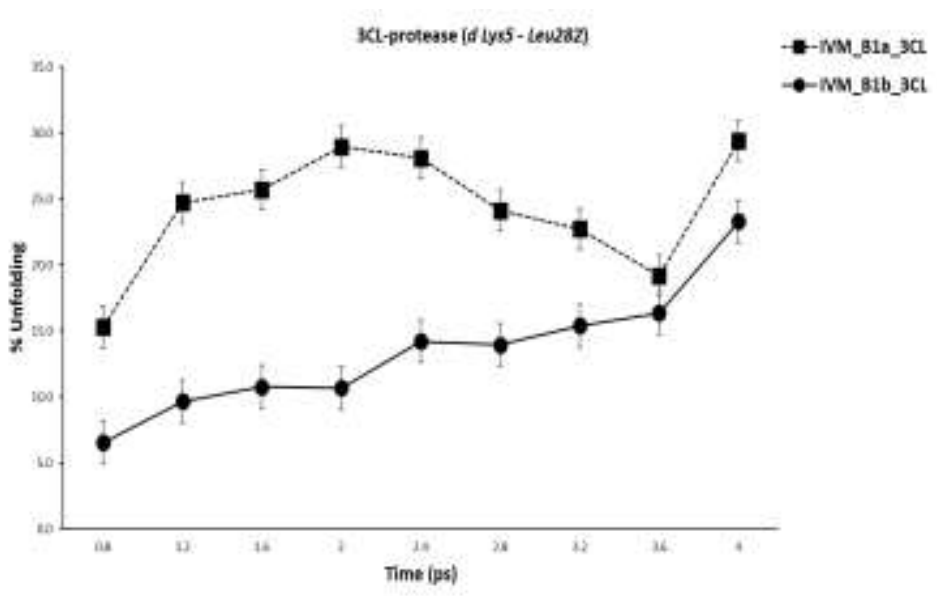

B

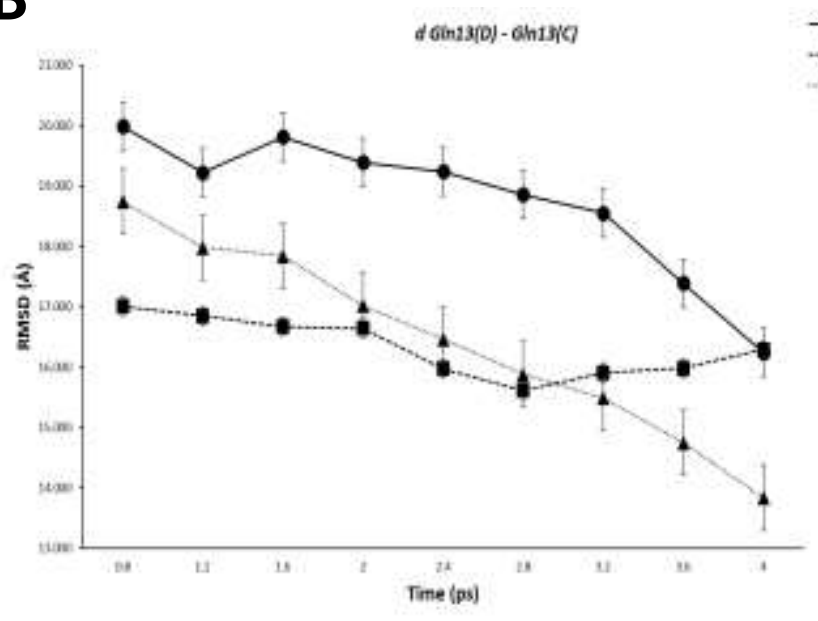

D

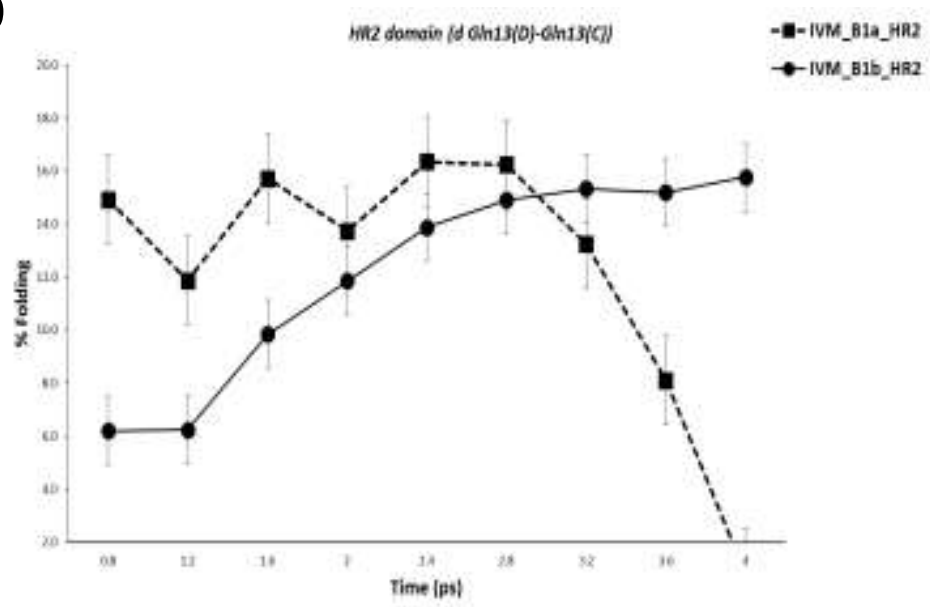

Figure 4. Conformational fluctuation and partial unfolding or folding of 3CL-protease (A and C) and HR2 domain (B and D) in presence of ivermectin B1a_CID_6321424 and ivermectin B1b_CID_6321425 as a function of time. Using as a reference the distances between the residues of Lys5 and Leu282, and Gln13(chain d) and Gln13(chain c) for 3CL-protease and HR2 domain, respectively. Arbitrarily chosen because they are at a distance of approximately 3-4 Å from the ligands. 
The results obtained in this work are promising because they show the existence of FDA approved drugs that are on the World Health Organization's List of Essential Drugs (WHO, 2019) and that could be used as models in more exhaustive bioinformatics or experimental studies related to structure-activity studies to develop alternatives against SARS-VOC-2. This is very important because the active sites of the SARS coronavirus proteins have been reported to show important conformational differences and, therefore, the docking sites of SARS-COV-2 are not compatible, which could compromise the efficacy of reused drugs (Chang et al., 2020; Bzowka et al., 2020).

\section{Conclusion}

Based on the theoretical tools applied in this study, it was discovered that ivermectins B1a and B1b bind of different of manner to the 3CL protease and the HR2 domain of SARS-COV-2 and can promote important structural changes in these structures, inducing unfolding/folding of these viral proteins. We recommend that more experimental studies should carry out about molecular mechanism to establish the possible relationship of the results obtained in this work with the observed biological activity of this drug on SARS-COV-2.

\section{References}

Adem, S.; Eyupoglu, V.; Sarfraz, I.; Rasul, A.; Ali, M. 2020. Identification of Potent SARSCOV-2 Main Protease (Mpro) Inhibitors from Natural Polyphenols: An in Silico Strategy Unveils a Hope against CORONA. Preprints, 2020030333. 
Alamri, M.A.; Tahir ul Qamar, M.; Alqahtani, S.M. 2020. Pharmacoinformatics and Molecular Dynamic Simulation Studies Reveal Potential Inhibitors of SARS-CoV-2 Main Protease 3CLpro. Preprints, 2020020308.

Askin, S., Bond, T. E., Sorenson, A. E., Moreau, M. J., Antony, H., Davis, R. A., Schaeffer, P. M. 2018. Selective protein unfolding: A universal mechanism of action for the development of irreversible inhibitors. Chemical communications, 54(14), 1738-1741.

Bhal, S. 2007. LogP-Making sense of the value. Advanced chemistry development, Toronto, ON, Canada, 1-4.

Bzowka, M., Mitusinska, K., Raczynska, A., Samol, A., Tuszynski, J. A., Gora, A. 2020. Molecular Dynamics Simulations Indicate the SARS-COV-2 Mpro Is Not a Viable Target for Small-Molecule Inhibitors Design. bioRxiv.

Caly, L., Druce, J.D., Catton, M.G., Jans, D.A., Wagstaff, K.M. 2020. The FDA-approved Drug Ivermectin inhibits the replication of SARS-CoV-2 in vitro, Antiviral Research, https://doi.org/10.1016/j.antiviral.2020.104787.

Chandel, V., Raj, S., Rathi, B., Kumar, D. 2020. In Silico Identification of Potent SARS-COV-2 Main Protease Inhibitors from FDA Approved Antiviral Compounds and Active Phytochemicals through Molecular Docking: A Drug Repurposing Approach. doi:10.20944/preprints202003.0349.v1.

Chang, Y., Tung, Y., Lee, K., Chen, T., Hsiao, Y., Chang, H and Shih, S. 2020. Potential therapeutic agents for SARS-COV-2 based on the analysis of protease and RNA polymerase docking. preprints.org. 
Cheminformatics, M. 2020. Molinspiration. Web-enabled software for large-scale calculation of molecular properties and database searches, Free online molecular descriptor calculations.

Choudhary, S., Malik, Y. S., Tomar, S., Tomar, S. 2020. Identification of SARS-CoV-2 Cell Entry Inhibitors by Drug Repurposing Using in Silico Structure-Based Virtual Screening Approach. chemrxiv.org.

Coleman, C. M., Sisk, J. M., Mingo, R. M., Nelson, E. A., White, J. M., Frieman, M. B. 2016. Abelson kinase inhibitors are potent inhibitors of severe acute respiratory syndrome coronavirus and middle east respiratory syndrome coronavirus fusion. Journal of virology, 90(19), 8924-8933.

Contini, A. 2020. Virtual screening of an FDA approved drugs database on two SARS-COV-2 coronavirus proteins. chemrxiv.org.

Devaux, C., Rolain, J., Colson, P and Raoult, D. 2020. New insights on the antiviral effects of chloroquine against coronavirus: what to expect for SARS-COV-2?. International Journal of Antimicrobial Agents, 105938.

Daina, A., Michielin, O., Zoete, V. 2017. SwissADME: a free web tool to evaluate pharmacokinetics, drug-likeness and medicinal chemistry friendliness of small molecules. Scientific reports, $7,42717$.

Dos Santos, K., Guedes, I., Karl, A and Dardenne, L. 2020. Highly flexible ligand docking: Benchmarking of the DockThor program on the LEADS-PEP protein-peptide dataset. Journal of Chemical Information and Modeling. 60(2), 667-683. 
Elshabrawy, H. A., Coughlin, M. M., Baker, S. C., Prabhakar, B. S. 2012. Human monoclonal antibodies against highly conserved HR1 and HR2 domains of the SARS-CoV spike protein are more broadly neutralizing. Plos one, 7(11).

Gautret, P., Lagier, J., Parola, P., Meddeb, L., Mailhe, M., Doudier, B and Honoré, S. 2020. Hydroxychloroquine and azithromycin as a treatment of SARS-COV-2: results of an openlabel non-randomized clinical trial. International Journal of Antimicrobial Agents, 105949.

Gentile, D.; Patamia, V.; Scala, A.; Sciortino, M.T.; Piperno, A.; Rescifina, A. 2020. Inhibitors of SARS-CoV-2 Main Protease from a Library of Marine Natural Products: A Virtual Screening and Molecular Modeling Study. Preprints, 2020030372.

Gomez, D., Huber, K and Klumpp, S. 2019. On protein folding in crowded conditions. The Journal of Physical Chemistry Letters, 10(24), 7650-7656.

Gonzalez-Paz, L., Lossada, C., Moncayo, L., Romero, F., Paz, J., Vera-Villalobos, J., Pérez, A., San-Blas, E., Alvarado, Y. 2020. Theoretical Molecular Docking Study of the Structural Disruption of the Viral 3CL-Protease of COVID19 Induced by Binding of Capsaicin, Piperine and Curcumin Part 1: A Comparative Study with Chloroquine and Hydrochloroquine Two Antimalaric Drugs, PREPRINT (Version 1) available at Research Square, DOI:10.21203/rs.3.rs-21206/v1.

Jiang, F., Deng, L., Zhang, L., Cai, Y., Cheung, C and Xia, Z. 2020. Review of the clinical characteristics of coronavirus disease 2019 (SARS-COV-2). Journal of General Internal Medicine, 1-5. 
Kasahara, K., Ma, B., Goto, K., Dasgupta, B., Higo, J., Fukuda, I and Nakamura, H. 2016. myPresto/omegagene: a GPU-accelerated molecular dynamics simulator tailored for enhanced conformational sampling methods with a non-Ewald electrostatic scheme. Biophysics and Physicobiology, 13, 209-216.

Khaerunnisa, S.; Kurniawan, H.; Awaluddin, R.; Suhartati, S.; Soetjipto, S. 2020. Potential Inhibitor of SARS-COV-2 Main Protease (Mpro) From Several Medicinal Plant Compounds by Molecular Docking Study. Preprints, 2020030226.

Krähling, V., Stein, D. A., Spiegel, M., Weber, F., Mühlberger, E. 2009. Severe acute respiratory syndrome coronavirus triggers apoptosis via protein kinase $\mathrm{R}$ but is resistant to its antiviral activity. Journal of virology, 83(5), 2298-2309.

Liu, X., Zhang, B., Jin, Z., Yang, H and Rao, Z. 2020. The crystal structure of 2019-NCoV main protease in complex with an inhibitor N3. RCSB Protein Data Bank.

Mizutani, T., Fukushi, S., Saijo, M., Kurane, I., Morikawa, S. 2005. JNK and PI3k/Akt signaling pathways are required for establishing persistent SARS-CoV infection in Vero E6 cells. Biochimica et Biophysica Acta (BBA) - Molecular Basis of Disease, 1741(1-2): 4-10.

Mizutani, T., Fukushi, S., Saijo, M., Kurane, I., Morikawa, S. 2004. Importance of Akt signaling pathway for apoptosis in SARS-CoV-infected Vero E6 cells. Virology, 327(2): 169-174.

Mohammad, F., Mohsin, A., Zaw, A., Tanveer, A., Waseem, A. 2020. Identification of Dietary Molecules as Therapeutic Agents to Combat SARS-COV-2 Using Molecular Docking Studies, PREPRINT (Version 1) available at Research Square. 
Mastrangelo, E., Pezzullo, M., De Burghgraeve, T., Kaptein, S., Pastorino, B., Dallmeier, K., Lamballerie, X., Neyts, J., Hanson, A., Frick, D., Bolognesi, M., Milani, M. 2012. Ivermectin is a potent inhibitor of flavivirus replication specifically targeting NS3 helicase activity: new prospects for an old drug. Journal of Antimicrobial Chemotherapy, 67(8), 1884-1894.

Reena Roy, D., Kandagalla, S and Krishnappa, M. 2020. Exploring the ethnomycological potential of Lentinus squarrosulus Mont. through GC-MS and chemoinformatics tools. Mycology, 11(1), 78.

Sekhar, T. 2020. Virtual Screening based prediction of potential drugs for SARS-COV-2. Preprints, doi:10.20944/preprints202002.0418.v2.

Sharma, A.D.; Kaur, I. 2020. Eucalyptol (1,8 cineole) from Eucalyptus Essential Oil a Potential Inhibitor of COVID 19 Corona Virus Infection by Molecular Docking Studies. Preprints 2020030455.

Sun, N.; Wong, W.; Guo, J. 2020. Prediction of Potential 3CLpro-Targeting Anti-SARS-CoV-2 Compounds from Chinese Medicine. Preprints, 2020030247.

Thomsen, R and Christensen, M. 2006. MolDock: a new technique for high-accuracy molecular docking. Journal of Medicinal Chemistry, 49(11), 3315-3321.

Tong, T. R. 2009. Drug targets in severe acute respiratory syndrome (SARS) virus and other coronavirus infections. Infectious Disorders-Drug Targets (Formerly Current Drug TargetsInfectious Disorders), 9(2), 223-245. 
Qamar, M., Alqahtani, S., Alamri, M and Chen, L. 2020. Structural basis of SARS-CoV-2 3CLpro and anti-SARS-COV-2 drug discovery from medicinal plants. preprints.org.

Varghese, F., Kaukinen, P., Gläsker, S., Bespalov, M., Hanski, L., Wennerberg, K., Kümmerer, B., Ahola, T. 2016. "Discovery of berberine, abamectin and ivermectin as antivirals against chikungunya and other alphaviruses". Antiviral Research. 126: 117-24.

Velavan, T. P., Meyer, C. G. 2020. The COVID-19 epidemic. Trop Med Int Health, 25(3), 278280.

Wang, J. 2020. Fast Identification of possible drug treatment of coronavirus disease-19 (SARSCOV-2) through computational drug repurposing study. chemrxiv.org.

Wang, M., Cao, R., Zhang, L., Yang, X., Liu, J., Xu, M and Xiao, G. 2020. Remdesivir and chloroquine effectively inhibit the recently emerged novel coronavirus (2019-nCoV) in vitro. Cell Research, 30(3), 269-271.

World Health Organization (WHO), 2019. World Health Organization model list of essential medicines: 21st list 2019. Geneva: World Health Organization. hdl:10665/325771. WHO/MVP/EMP/IAU/2019.06. License: CC BY-NC-SA 3.0 IGO.

World Health Organization (WHO), 2020. Coronavirus disease 2019 (COVID-19): situation report, 76.

Xia, S., Liu, M., Wang, C., Xu, W., Lan, Q., Feng, S., ... \& Qin, C. 2020. Inhibition of SARSCoV-2 (previously 2019-nCoV) infection by a highly potent pan-coronavirus fusion inhibitor targeting its spike protein that harbors a high capacity to mediate membrane fusion. Cell Research, 1-13. 
Xu, X., Chen, P., Wang, J., Feng, J., Zhou, H., Li, X and Hao, P. 2020. Evolution of the novel coronavirus from the ongoing Wuhan outbreak and modeling of its spike protein for risk of human transmission. Science China Life Sciences, 63(3), 457-460.

Yazwinski, T., Williams, M., Greenway, T., Tilley, W. 1981. Anthelmintic activities of ivermectin against gastrointestinal nematodes of cattle. American Journal of Veterinary Research, 42(3):481-482.

Zhang, J., Shen, X., Yan, Y. M., Yan, W. A. N. G., Cheng, Y. X. 2020. Discovery of anti-SARSCoV-2 agents from commercially available flavor via docking screening. osf.io.

Zoete, V., Daina, A., Bovigny, C., Michielin, O. 2016. SwissSimilarity: A Web Tool for Low to Ultra High Throughput Ligand-Based Virtual Screening., J. Chem. Inf. Model., 56(8), 13991404. 